\title{
Arrhythmia in Pregnancy
}

\author{
Chennapragada Sridevi ${ }^{1}$ \\ ${ }^{1}$ Care Hospitals and Care Foundation, Hyderabad, Telangana, India
}

\begin{abstract}
Address for correspondence Chennapragada Sridevi, DNB (Medicine), DNB (Cardiology), MNAMS, CCDS, Care Hospitals, Road No. 10, Banjara Hills, Hyderabad 500034, Telangana, India (e-mail: dr_csridevi@yahoo.com).
\end{abstract}

Indian J Cardiovasc Dis Women-WINCARS 2018;3:132-138
Abstract
Keywords
- atrial fibrillation
- bradycardia
- cardiac arrest
- DC cardioversion
- intracardiac echocardiography
- oral anticoagulation
- supraventricular tachycardia
- ventricular tachycardia

Arrhythmias in pregnancy are uncommon. They are associated with significant hemodynamic burden on a pregnant mother. Tachycardia that induces hemodynamic instability affects the fetus and neonate apart from the mother. In this review, a brief attempt is made to discuss various arrhythmias that occur in pregnancy and their management.

\section{Introduction}

Arrhythmias may occur during pregnancy for the first time or can be a manifestation of previous existing arrhythmia. In general, management of arrhythmias depends on the drugs used. There are no randomized controlled studies that are done to show efficacy and safety of these drugs on fetus or neonate. Most arrhythmias can be treated conservatively. Few patients require careful consideration and judicious use of antiarrhythmic drugs (AADs), DC cardioversion, and device management. A collaborative team of the gynecologist and neonatologist with the cardiologist will yield better outcomes.

\section{Arrhythmogenesis}

There are multiple hypotheses for development of arrhythmias in pregnancy. They are structural changes, hormonal, and sympathetic stimulation.

\section{Structural Changes}

Pregnancy is associated with increased plasma volume from 8 weeks of pregnancy and reaches a peak around 36 weeks.
It has been noted that atrial and ventricular myocytes get stretched due to increase in plasma volume, resulting in early after depolarizations, shortened refractoriness, slowed conduction, and spatial dispersion through activation of stretch-activated ion channels. ${ }^{1,2}$

\section{Hormones}

Hormonal and autonomic changes are some of factors that can cause arrhythmogenesis. Animal studies have shown drugs such as estradiol and progesterone to be proarrhythmic. In a report by Mahija et al, a woman had ventricular tachycardia (VT) during pregnancy, which was managed with drugs. In the postpartum period, she was taken for ablation, but VT could not be induced in electrophysiology (EP) laboratory. She was given hormones, and VT was successfully induced and ablated. ${ }^{3,4}$

\section{Sympathetic Stimulation}

In addition, estrogen-induced adrenergic responsiveness seems to be increased in pregnancy due to increase in adrenergic receptors. ${ }^{5,6}$ 


\section{Incidence of Arrhythmia}

Palpitation is a common symptom in pregnancy. Atrial ectopic beats occur, which generally require reassurance. Severe symptomatic arrhythmias are uncommon, that is, 2 to 3 in 100,000 cases. Incidence of sinus tachycardia is common., ${ }^{7}$ Significant sinus bradycardia does not occur. Bradycardia events occur due to congenital heart block, which are rare, that is, 1 in 20,000 cases. ${ }^{910}$

\section{History Taking}

Assessment of symptoms, degree of severity length, and timing are important. It is important to assess symptoms to weigh the risk and benefit to the mother and fetus as most of the antiarrhythmic agents are classified as class $\mathrm{C}$ (the drugs on which there are no human studies, about which the animal studies did not establish safety, or were harmful). Arrhythmias causing hemodynamic instability should be carefully and promptly assessed as they can cause fetal distress.

\section{Arrhythmia in Structurally Normal Heart}

Women with structurally normal heart present either first time with arrhythmia or with preexisting history of arrhythmia. Ectopic beats associated with prolonged reentry circuits precipitate arrhythmia. Women with previous history of arrhythmias are at a higher risk of having cardiac arrhythmias during pregnancy. A retrospective study of 73 patients with prior history of arrhythmias revealed that $44 \%$ of them with normal sinus rhythm at the beginning of their pregnancy developed a recurrence of their known tachyarrhythmia during or within 1 month after their pregnancy. ${ }^{11}$ The recurrence rate of supraventricular tachycardia (SVT), paroxysmal atrial fibrillation/atrial flutter (AF/AFL), and VT in women with a history of these arrhythmias was 50,52 , and $27 \%$, respectively. Women admitted with no known arrhythmia and no structural heart disease should be evaluated for hyperthyroidism and electrolyte imbalance. Hyperthyroidism causes AF during pregnancy. Wide QRS tachycardia can be SVT with aberrancy, preexcited tachycardia, or VT. VT arising from a structurally normal heart will be mostly arising from right ventricular outflow tract (RVOT). A recent study found $73 \%$ of the complex ventricular ectopy originating in the RVOT during pregnancy. ${ }^{12} \mathrm{~A}$ specific type of polymorphic VT, torsade de pointes, occurs in patients with long QT syndrome. A retrospective study of women affected with congenital long QT syndrome, who had one or more pregnancies, showed no increase in cardiac events during pregnancy but a definite rise in the ventricular arrhythmias during the postpartum period, especially in LQT2 genotype. ${ }^{13}$

\section{Women with Structural Heart Disease}

Survival of women with postoperative congenital heart disease has improved resulting in their reaching adulthood and becoming pregnant. The incidence of arrhythmia is increasing in women with rheumatic heart disease, and patients with postoperative valve replacement tend to have arrhythmia. Patients with cardiomyopathy, especially those with peripartum cardiomyopathy, have VT. A patient with mitral valve prolapse received an implantable cardioverter-defibrillator (ICD) for VT though it is a very rare. ${ }^{14}$ Focal intra-atrial reentry, AFL, and fibrillation are common in patients with postoperative atrial defects, rheumatic valve disease, and congestive cardiomyopathy. Focal atrial tachycardia (AT) occurs in $4.6 \%$ of unrepaired atrial septal defect (ASD) and 3.6\% of repaired ASD. ${ }^{15}$ Postoperative Fontan atrial switch can present with AT due to atrial reentry. Postoperative tetralogy and pulmonic stenosis can present with symptomatic atrial tachyarrhythmia. In a series of 45 women with postoperative Fontan surgery, 71 pregnancies were detected, and of those 71 pregnancies, 33 were complicated by primary atrial arrhythmia resulting in heart failure. ${ }^{16}$ Pregnant women with $\mathrm{AF}$, especially mitral valve disease, require pharmacologic rate control. In lesions such as left ventricular outflow tract (LVOT) obstruction and hypertrophic cardiomyopathy sinus, DC cardioversion can be done.

\section{Management of Specific Arrhythmias}

\section{Palpitations and Premature Beats}

In a study conducted in pregnant women without structural heart disease, premature atrial contractions (PACs) were $57 \%$ and premature ventricular contractions (PVCs) were $50 \%$. Exaggerated symptoms occur due to increase in cardiac volume and contractility. Reassurance is sufficient, but if the patient is significantly symptomatic, cardioselective $\beta$-blocker can be given preferably after the first trimester. ${ }^{8}$

\section{Supraventricular Arrhythmias}

Supraventricular tachycardia is the most common arrhythmia during pregnancy. Incidence of atrioventricular nodal reentrant tachycardia (AVNRT) is more than atrioventricular reentrant tachycardia (AVRT). ${ }^{17}$ Approximately $20 \%$ of patients present with recurrence of arrhythmia.

\section{Atrioventricular Nodal Reentrant Tachycardia}

Atrioventricular nodal reentrant tachycardia is one of the most common arrhythmias to be present in pregnancy. Most women who present with AVNRT have a structurally normal heart. If arrhythmia causes hemodynamic instability, that can result in fetal distress. AVNRT can be managed by avoiding precipitating factors and vagal maneuvers. If arrhythmia is persisting, adenosine is the drug of choice. Adenosine converts arrhythmia $95 \%$ of times, and if it does not, metoprolol or propranolol can be used. Verapamil is third line of choice. ${ }^{18}$ Long-term arrhythmia control can be achieved with $\beta$-blockers, or verapamil can be used. Sotalol or flecainide are the drugs of choice if the AV nodal-blocking drugs fail to control arrhythmia. Digoxin cannot be used alone. It can be combined with a $\beta$-blocker. Catheter ablation has been reported in cases of recurrent arrhythmia. ${ }^{19,20}$

\section{Atrioventricular Reentrant Tachycardia}

Many patients present with orthodromic tachycardia. The management is similar to patients presenting with 
AVNRT. In women presenting with wide complex tachycardia irregular in nature and manifest preexcitation, procainamide is the drug of choice. Long-term management $\beta$-blockers, calcium channel blockers, digoxin, and flecainide are used for concealed accessory pathway. Class 1 drugs such as flecainide and quinidine can be used to slow conduction across the pathway for patients with manifest pathway. Catheter ablation is preferred choice in nonpregnant women, but there are reports of successful ablation in symptomatic pregnant women. ${ }^{21}$

\section{Atrial Tachycardia}

Atrial tachycardia can occur and may persist during pregnancy. It may also present as tachy cardiomyopathy. AT is generally resistant to drug therapy and cardioversion. Digoxin, $\beta$-blockers, and diltiazem are drugs of choice. If not controlled by these drugs, flecainide or sotalol can be used. In cases of persistent drug refractory arrhythmia, there are reports of successful ablation of AT. ${ }^{18}$ Radiofrequency ablation is advised in hemodynamically unstable women or in women with recurrent palpitation in spite of medication. Ablation can be done without using fluoroscopy. Ferguson et al reported a successful ablation of left AT in a 27-year-old pregnant woman in whom intracardiac echocardiography (ICE) and electroanatomical mapping were used for the procedure with no fluoroscopy. ${ }^{21}$

\section{Atrial Flutter and Fibrillation}

Atrial fibrillation and atrial flutter are uncommon during pregnancy with an incidence of 2 in $100,000 .{ }^{22} \mathrm{AF}$ is associated with structural heart disease, and pregnant women with previous history are more prone to episodes of AF during their pregnancy period. For a new-onset AF during pregnancy, evaluation of pulmonary embolism should be done. Ibutilide can be used for acute episode of AF during pregnancy. Digoxin, calcium blocker, and $\beta$-blocker can be used for acute and chronic therapy. Dronedarone and dofetilide are contraindicated. Alternatively, sotalol or ibutilide can be used. Amiodarone causes fetal harm; hence, it is used only for life-threatening instances where catheter ablation is not indicated. Cavotricuspid isthmus ablation can be done in AFL, but there are no reports.

\section{Systemic Anticoagulation}

Aspirin can be given during pregnancy. In pregnant women requiring oral anticoagulant (OAC), Warfarin can be given in the second trimester. Warfarin is contraindicated in the first and third trimesters. Warfarin is replaced with lowmolecular-weight heparin (LMWH) or unfractionated heparin (UFH). UFH may cause heparin-induced thrombocytopenia. ${ }^{22}$

\section{Ventricular Tachycardia and Sudden Cardiac Death}

Studies show that 2 in 100,000 women present with VT during pregnancy. ${ }^{23}$ It is more commonly found in pregnant women with structural heart disease and having previous history with recurrence rates being as high as $27 \% .{ }^{11}$ Pregnant women with hypertrophic cardiomyopathy and arrhythmogenic right ventricular dysplasia (ARVD) present with
VT in later weeks of pregnancy or early postpartum period peripartum cardiomyopathy and needs further evaluation. ${ }^{24}$ Patients with congenital heart disease, postoperative repair presents with VT more frequently from 4.5 to $15.9 \% .{ }^{25}$ Ischemic cardiomyopathy is rare in this age group, but patients can present with VT secondary to coronary spasm or spontaneous dissection. ${ }^{26}$ In women with hemodynamically stable VT, lignocaine can be tried. If lignocaine is not converted, procainamide or quinidine can be tried. Women with hemodynamically unstable VT require cardioversion. For chronic therapy, AAD with metoprolol or propranolol can be started. Patients nonresponsive to $\beta$-blockers, sotalol can be tried. Alternative drugs such as mexiletine and quinidine can be tried. VT ablation, unlike SVT ablation, requires prolonged procedure time longer fluoroscopy, and general anesthesia. There is only one case report of VT ablation. In a structurally normal heart, VT can manifest as idiopathic VT or long QT syndrome.

\section{Management of Idiopathic Ventricular Tachycardia}

Idiopathic VT arises from RVOT. $\beta$-Blockers are effective. If patients are nonresponsive to $\beta$-blocker, sotalol can be used. Verapamil is found to be sensitive for treating fascicular VT. Pregnant women receiving ablation for idiopathic VT has not been reported. - Fig. 1 shows a 24-year-old women (primigravida) presenting with idiopathic VT. - Fig. 2 shows the ECG of the same patient presenting with ventricular bigeminy after being administered with $\beta$-blockers.

\section{Long QT Syndrome}

The QT interval shortens during pregnancy; hence, events are much less during pregnancy. In postpartum period, decreases in heart rate altered sleep pattern stress are associated with increase in QT interval. Another retrospective study revealed 111 probands in 422 women, and they reported with having long QT. Women with probands are more susceptible to having syncope and aborted cardiac arrest. Sudden cardiac deaths were more in the postpartum period than ante partum period (23.4\% vs. $3.8 \%)^{27}$

\section{Cardioversion}

DC cardioversion can be done for hemodynamically unstable arrhythmias during any time of pregnancy. There is no or very minimal chance of fetal arrhythmia. During the third trimester, premature labor can be induced. ${ }^{28,29}$

\section{Implantable Cardioverter-Defibrillator and Pregnancy}

Pregnant women presenting with unstable VT and showing indication for ICD should go ahead with ICD implant. ICD can be implanted with minimal fluoroscopy or echocardiogram guidance. In a study by Natale et al, it was observed that 44 of the pregnant women had ICDs of which abdominal generators were used in 42 . Of the 44,11 (25\%) of the women received more than one shock without affecting the pregnancy. Further studies also revealed that pregnancy was not associated with an increase in ICD shocks. ${ }^{30}$ In a series by Boulé, 12 women with ICDs had 20 pregnancies and 7 of the 12 women had cardiomyopathy whereas 5 had channelopathies. The study 


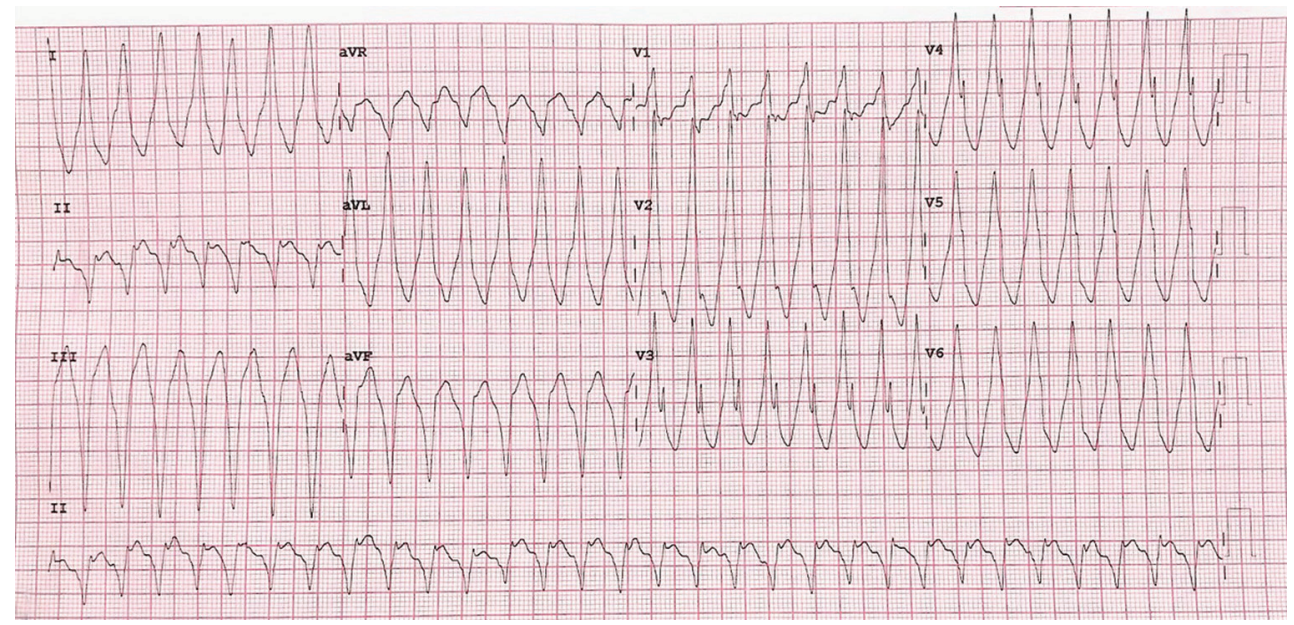

Fig. 1 ECG showing idiopathic ventricular tachycardia.

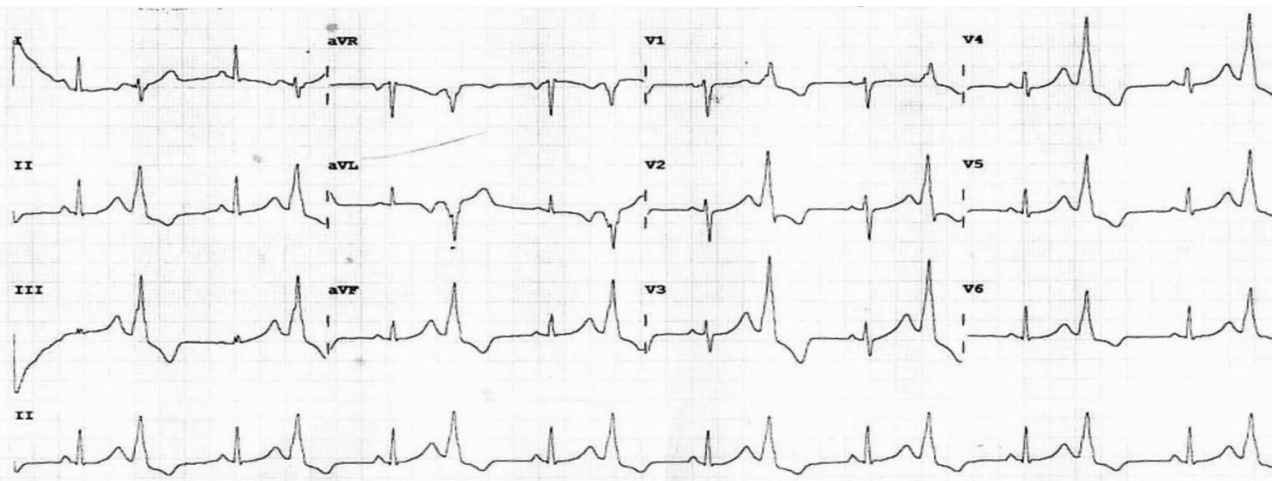

Fig. 2 ECG showing ventricular bigeminy after administration of $\beta$-blockers.

reported no maternal deaths and 14 live births. Two women in the study were given 12 shocks, and 1 woman reported of a miscarriage after a 4-week gestation period. The study also revealed one stillbirth in a woman with long QT syndrome and three miscarriages in a woman with repaired tetralogy of Fallot and one termination in repaired Laubry-Pezzi syndrome. It was observed that ICD operation had no effect on pregnancy. The continuation of $\beta$-blocker therapy for all women carrying ICDs in pregnancy is more beneficial than the risks. ${ }^{31}$

\section{Brady Arrhythmia}

Perloff described that the incidence of complete heart block (CHB) was estimated to be 1 in 15,000 to 20,000 live births. ${ }^{32}$ It may be congenital or degenerative. Pregnant women present rarely with degenerative $\mathrm{CHB}$ as it occurs after 50 years of age. ${ }^{33}$ However, congenital variety is seen during pregnancy, but that is also very rare and only few cases have been reported in the literature. Approximately $13 \%$ of patients undergoing cesarean section under spinal anesthesia can present with all grades of heart block. ${ }^{34,35}$ Pacing may not be required in women with physiologic bradycardia. ${ }^{36}$ For patients requiring permanent pacing during pregnancy, there are reports of pacing done with guidance of ECG, echocardiography, and ICE. ${ }^{37}$ Patients on permanent pacemaker undergo successful deliveries. ${ }^{38,39}$ - Fig. 3 shows the ECG of a patient having a complete heart block with a narrow escape at the rate of 35 beats/min. - Fig. 4 shows the ECG of the same patient who had a pacemaker implantation and then had a successful delivery through cesarean section.

\section{Bradycardia Associated with anti-Ro/SSA Antibodies}

There are two possible forms of anti-Ro/SSA-associated atrioventricular block (AVB) in adults: one is an acquired form and the other is late progressive congenital form. The conduction defect in the acquired form is associated with the electrophysiologic inhibition of calcium channels on cardiomyocytes. ${ }^{40}$

\section{Cardiac Arrest in Pregnant Women}

Mhyre et al, in a series of hospitalizations for delivery between 1998 and 2011, described that in 1 in 12,000 cardiac arrests (or 8.5 per 100,000 ), the survival was only $59 \%{ }^{41}$ Resuscitation in pregnant women is peculiar as there are two lives to be considered. It has to be a team effort between medical, obstetrical, and neonatology services. The mother has to be resuscitated in supine position with manual tilt of the abdomen to left lateral position. Cesarean section should be initiated if the pregnant woman has a cardiac arrest and is not resuscitated in 4 minutes. ${ }^{42}$

\section{Arrhythmia in Pregnancy: European Society of Cardiology Guidelines 2018}

Burden of arrhythmia is increasing in pregnancy. Some of the important factors include women having late pregnancies 


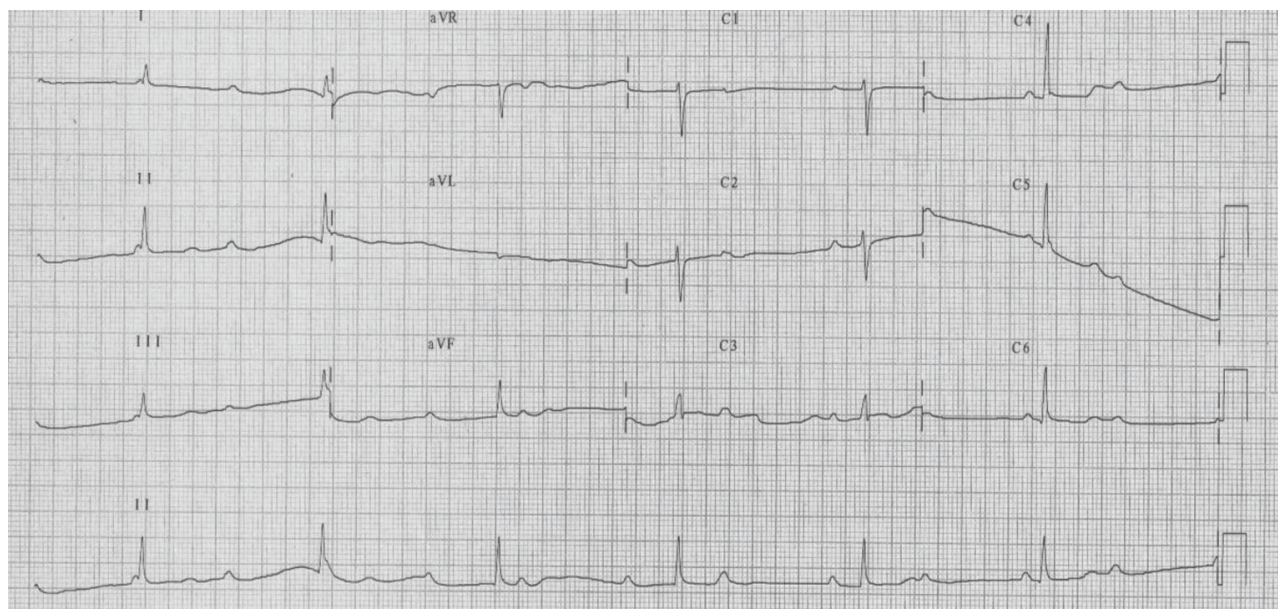

Fig. 3 Complete heart block with a narrow escape at the rate of 35 beats $/ \mathrm{min}$.

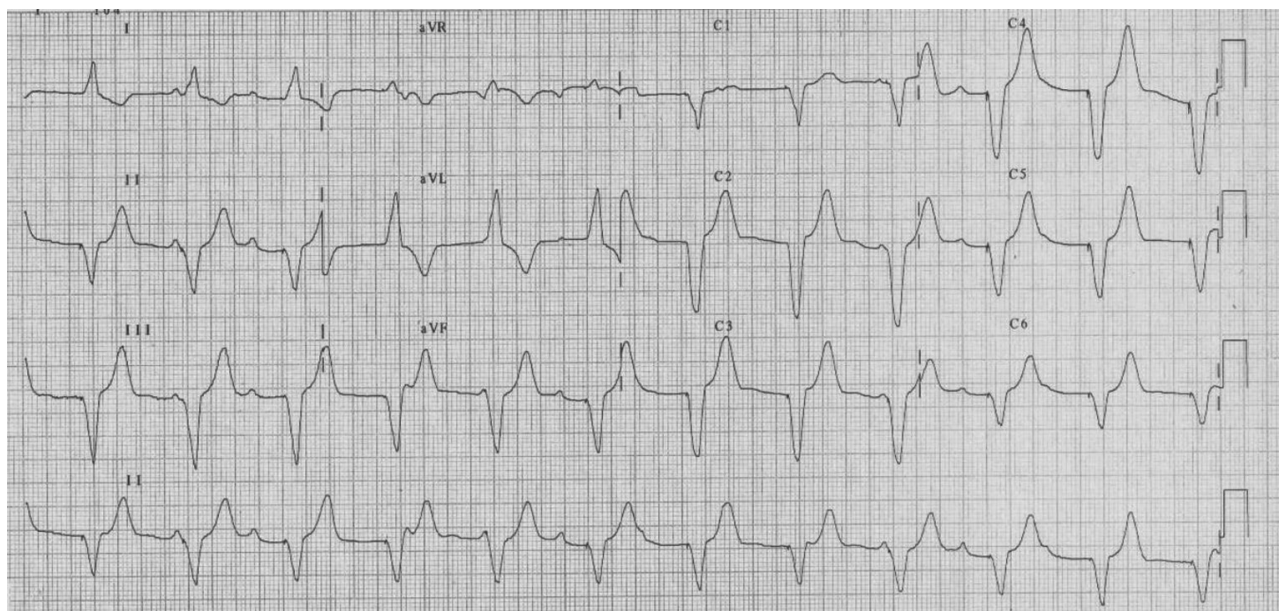

Fig. 4 ECG after pacemaker implantation.

and increasing incidence of women surviving to higher age and bearing pregnancy. This article validates all the recommendations made in The European Society of Cardiology Guidelines 2018. ${ }^{43}$

\section{Conclusion}

The diagnostic criteria for arrhythmia have not been changed in pregnancy, but it is important that previous history of arrhythmias is well documented to ensure a good management plan. Drugs used to maintain rhythms and hemodynamic stability should be used conservatively, and a sustained arrhythmia requires a complete evaluation of the cardiac anatomy. Anatomical abnormalities require quick and comprehensive evaluation for management of arrhythmias, as rhythms vary depending on these abnormalities. A multidisciplinary approach of having cardiologists, gynecologists, and neonatologists in case of concern about fetal distress can influence treatment decisions and must be implemented. Radiation-related investigations need to be avoided as much as possible, but if essential, adequate measures to protect the abdomen area with lead shield must be done. Ablation with newer technologies such as ICE and three-dimensional (3D) anatomic mapping avoids radiation-related issues.

\section{Acknowledgments}

The author acknowledges the contribution of Dr. Rajeev Menon, Consultant Cardiologist and Medical Director, CARE Hitech Hospital, for providing valuable insights in the management of arrhythmias in pregnant women. Due appreciation is given to CARE Foundation for helping the author in the compilation of this article.

\section{References}

1 Franz MR, Cima R, Wang D, Profitt D, Kurz R. Electrophysiological effects of myocardial stretch and mechanical determinants of stretch-activated arrhythmias. Circulation 1992;86(3):968-978

2 Ninio DM, Saint DA. The role of stretch-activated channels in atrial fibrillation and the impact of intracellular acidosis. Prog Biophys Mol Biol 2008;97(2-3):401-416

3 Odening KE, Choi BR, Liu GX, et al. Estradiol promotes sudden cardiac death in transgenic long QT type 2 rabbits while progesterone is protective. Heart Rhythm 2012;9(5):823-832 
4 Makhija A, Sharada K, Hygriv Rao B, Thachil A, Narsimhan C. Hormone sensitive idiopathic ventricular tachycardia associated with pregnancy: successful induction with progesterone and radiofrequency ablation. J Cardiovasc Electrophysiol 2011;22(1):95-98

5 Roberts JM, Insel PA, Goldfien A. Regulation of myometrial adrenoreceptors and adrenergic response by sex steroids. Mol Pharmacol 1981;20(1):52-58

6 Barron WM, Mujais SK, Zinaman M, Bravo EL, Lindheimer MD. Plasma catecholamine responses to physiologic stimuli in normal human pregnancy. Am J Obstet Gynecol 1986;154(1):80-84

7 Blomström-Lundqvist C, Scheinman MM, Aliot EM, et al; American College of Cardiology; American Heart Association Task Force on Practice Guidelines; European Society of Cardiology Committee for Practice Guidelines. Writing Committee to Develop Guidelines for the Management of Patients With Supraventricular Arrhythmias. ACC/AHA/ESC guidelines for the management of patients with supraventricular arrhythmias. Circulation 2003;108(15):1871-1909

8 Shotan A, Ostrzega E, Mehra A, Johnson JV, Elkayam U. Incidence of arrhythmias in normal pregnancy and relation to palpitations, dizziness, and syncope. Am J Cardiol 1997;79(8):1061-1064

9 Gowda RM, Khan IA, Mehta NJ, Vasavada BC, Sacchi TJ. Cardiac arrhythmias in pregnancy: clinical and therapeutic considerations. Int J Cardiol 2003;88(2-3):129-133

10 Michaëlsson $\mathrm{M}$, Jonzon $\mathrm{A}$, Riesenfeld $\mathrm{T}$. Isolated congenital complete atrioventricular block in adult life. A prospective study. Circulation 1995;92(3):442-449

11 Silversides CK, Harris L, Haberer K, Sermer M, Colman JM, Siu SC. Recurrence rates of arrhythmias during pregnancy in women with previous tachyarrhythmia and impact on fetal and neonatal outcomes. Am J Cardiol 2006;97(8):1206-1212

12 Brodsky M, Doria R, Allen B, Sato D, Thomas G, Sada M. New-onset ventricular tachycardia during pregnancy. Am Heart J 1992;123(4 Pt 1):933-941

13 Seth R, Moss AJ, McNitt S, et al. Long QT syndrome and pregnancy. J Am Coll Cardiol 2007;49(10):1092-1098

14 Saha T, Norris R, Luebbert J. Recurrent premature ventricular contraction-induced ventricular fibrillation and resuscitated sudden death in a 26-year-old pregnant woman with bileaflet mitral valve prolapse. HeartRhythm Case Rep 2017;4(2):58-62

15 Yap SC, Drenthen W, Meijboom FJ, et al; ZAHARA investigators. Comparison of pregnancy outcomes in women with repaired versus unrepaired atrial septal defect. BJOG 2009;116(12):1593-1601

16 Chugh R. Management of pregnancy in women with repaired CHD or after the Fontan procedure. Curr Treat Options Cardiovasc Med 2013;15(5):646-662

17 Lee SH, Chen SA, Wu TJ, et al. Effects of pregnancy on first onset and symptoms of paroxysmal supraventricular tachycardia. Am J Cardiol 1995;76(10):675-678

18 Szumowski L, Szufladowicz E, Orczykowski M, et al. Ablation of severe drug-resistant tachyarrhythmia during pregnancy. J Cardiovasc Electrophysiol 2010;21(8):877-882

19 Bongiorni MG, Di Cori A, Soldati E, et al. Radiofrequency catheter ablation of atrioventricular nodal reciprocating tachycardia using intracardiac echocardiography in pregnancy. Europace 2008;10(8):1018-1021

20 Kanjwal Y, Kosinski D, Kanj M, Thomas W, Grubb B. Successful radiofrequency catheter ablation of left lateral accessory pathway using transseptal approach during pregnancy. J Interv Card Electrophysiol 2005;13(3):239-242

21 Ferguson JD, Helms A, Mangrum JM, DiMarco JP. Ablation of incessant left atrial tachycardia without fluoroscopy in a pregnant woman. J Cardiovasc Electrophysiol 2011;22(3):346-349

22 January CT, Wann LS, Alpert JS, et al; ACC/AHA Task Force Members. 2014 AHA/ACC/HRS guideline for the management of patients with atrial fibrillation: executive summary: a report of the American College of Cardiology/American Heart Association Task Force on practice guidelines and the Heart Rhythm Society. Circulation 2014;130(23):2071-2104 http:// content.onlinejacc.org/

23 Li JM, Nguyen C, Joglar JA, Hamdan MH, Page RL. Frequency and outcome of arrhythmias complicating admission during pregnancy: experience from a high-volume and ethnicallydiverse obstetric service. Clin Cardiol 2008;31(11):538-541

24 Puri A, Sethi R, Singh B, et al. Peripartum cardiomyopathy presenting with ventricular tachycardia: a rare presentation. Indian Pacing Electrophysiol J 2009;9(3):186-189

25 Tateno S, Niwa K, Nakazawa M, Akagi T, Shinohara T, Yasuda T; Study Group for Arrhythmia Late after Surgery for Congenital Heart Disease (ALTAS-CHD). Arrhythmia and conduction disturbances in patients with congenital heart disease during pregnancy: multicenter study. Circ J 2003;67(12):992-997

26 Hankins GD, Wendel GD Jr., Leveno KJ, Stoneham J. Myocardial infarction during pregnancy: a review. Obstet Gynecol 1985;65(1):139-146

27 Rashba EJ, Zareba W, Moss AJ, et al; LQTS Investigators. Influence of pregnancy on the risk for cardiac events in patients with hereditary long QT syndrome. Circulation 1998;97(5):451-456

28 Wang YC, Chen $\mathrm{CH}$, Su HY, Yu MH. The impact of maternal cardioversion on fetal haemodynamics. Eur J Obstet Gynecol Reprod Biol 2006;126(2):268-269

29 Barnes EJ, Eben F, Patterson D. Direct current cardioversion during pregnancy should be performed with facilities available for fetal monitoring and emergency caesarean section. BJOG 2002;109(12):1406-1407

30 Natale A, Davidson T, Geiger MJ, Newby K. Implantable cardioverter-defibrillators and pregnancy: a safe combination? Circulation 1997;96(9):2808-2812

31 Boulé S, Ovart L, Marquié C. Pregnancy in women with an implantable cardioverter-defibrillator: is it safe? Europace 2014;16:1587-1594

32 Perloff JK. The Clinical Recognition of Congenital Heart Disease. 6th ed. Philadelphia, PA: Elsevier; 2003

33 Suri V, Keepanasseril A, Aggarwal N, Vijayvergiya R, Chopra S, Rohilla M. Maternal complete heart block in pregnancy: analysis of four cases and review of management. J Obstet Gynaecol Res 2009;35(3):434-437

34 Mandal S, Mandal D, Sarkar A, et al. Complete heart block and pregnancy outcome: an analysis from eastern India. SOJ Gynaecol Obstet Womens Health. 2015;1(1):5

35 Shen CL, Ho YY, Hung YC, Chen PL. Arrhythmias during spinal anesthesia for cesarean section. Can J Anaesth 2000;47(5):393-397

36 Hidaka N, Chiba Y, Fukushima K, Wake N. Pregnant women with complete atrioventricular block: perinatal risks and review of management. Pacing Clin Electrophysiol 2011;34(9):1161-1176

37 Güdal M, Kervancioğlu C, Oral D, Gürel T, Erol C, Sonel A. Permanent pacemaker implantation in a pregnant woman with the guidance of ECG and two-dimensional echocardiography. Pacing Clin Electrophysiol 1987;10(3 Pt 1):543-545

38 Sharma JB, Malhotra M, Pundir P. Successful pregnancy outcome with cardiac pacemaker after complete heart block. Int J Gynaecol Obstet 2000;68(2):145-146

39 Dalvi BV, Chaudhuri A, Kulkarni HL, Kale PA. Therapeutic guidelines for congenital complete heart block presenting in pregnancy. Obstet Gynecol 1992;79(5 (Pt 2)):802-804

40 Lazzerini PE, Brucato A, Capecchi PL, et al. Isolated atrioventricular block of unknown origin in the adult and autoimmunity: diagnostic and therapeutic considerations exemplified by 3 anti-Ro/SSA-associated cases. HeartRhythm Case Rep 2015;1(5):293-299 
41 Mhyre JM, Tsen LC, Einav S, Kuklina EV, Leffert LR, Bateman BT. Cardiac arrest during hospitalization for delivery in the United States, 1998-2011. Anesthesiology 2014;120(4):810-818

42 Jeejeebhoy, FM, Zelop, CM, Windrim, R, Carvalho, JC, Dorian, P, Morrison, LJ. Management of cardiac arrest in pregnancy: a systematic review. Resuscitation 2011;82:801-809
43 Regitz-Zagrosek V, Roos-Hesselink JW, Bauersachs J, et al; ESC Scientific Document Group. 2018 ESC guidelines for the management of cardiovascular diseases during pregnancy. Eur Heart J 2018;39(34):3165-3241 